\title{
Off-center dark matter halo leading to strong central disk lopsidedness
}

\author{
Chaitanya Prasad ${ }^{1}$ and Chanda J. $\operatorname{Jog}^{2}$ \\ 1 Department of Physics, Indian Institute of Technology Bombay, Mumbai 400076, India \\ e-mail: prasadchatty@phys.iitb.ac.in \\ 2 Department of Physics, Indian Institute of Science, Bangalore 560012, India \\ e-mail: cjjog@physics.iisc.ernet.in
}

Received 16 November 2016 / Accepted 6 January 2017

\begin{abstract}
There is increasing evidence from simulations and observations that the center of the dark matter halo in a Milky Way-type galaxy could be off-center by a few $100 \mathrm{pc}$ with respect to the galactic disk. We study the effect of such an offset halo on the orbits and kinematics in the central few kpc of the disk via a simple, analytical model. The equations of motion in the disk plane can be written in terms of the potentials of the disk and halo when they are concentric, and a perturbation term arising due to the offset halo. We show that this perturbation potential has an $m=1$ azimuthal variation, or is lopsided, and its magnitude increases at small radii. On solving these equations, we find that the perturbed orbit shows a large deviation, $\sim 40 \%$ in radius at $R=1.5 \mathrm{kpc}$, and also strong kinematical lopsidedness. Thus, even a small halo offset of $350 \mathrm{pc}$ can induce surprisingly strong spatial and kinematical lopsidedness in the central region within a $\sim 3 \mathrm{kpc}$ radius. The disk lopsidedness would have important implications for the evolution of this region; for example, it could help fuel the central active galactic nucleus.
\end{abstract}

Key words. galaxies: halos - galaxies: kinematics and dynamics - galaxies: spiral - galaxies: structure - Galaxy: center

\section{Introduction}

It is well known that a galactic disk is located within an extended dark matter halo, whose existence is deduced from a nearly flat rotation curve (Rubin 1983; Binney \& Tremaine 1987). The dark matter halo dominates the galaxy mass and hence the disk dynamics in the outer regions of a spiral galaxy. In the inner central region of $\sim 5 \mathrm{kpc}$ radius, however, the disk dominates the dynamics as it does in the Milky Way (Sackett 1997; Klypin et al. 2002). In theoretical as well as observational work, the dark matter halo is generally assumed to be concentric with respect to the disk for simplicity.

There is increasing evidence that the density peak of the dark matter halo of a Milky Way-type galaxy may be located off-center with respect to the disk center, as seen in the simulations (Kuhlen et al. 2013) where the typical separation is $300-400 \mathrm{pc}$. Once it is generated, the offset feature seems to be long-lived, lasting for several Gyr. Such an offset is of interest because it could provide a dark matter explanation of the peak in the $130 \mathrm{GeV}$ in the gamma-ray line emission that is displaced by about $200 \mathrm{pc}$ from Sgr A* in the Galactic plane (Kuhlen et al. 2013). There is also observational evidence for the halo being off-center with respect to the disk in a field galaxy, M 99 (Chemin et al. 2016), and also for a galaxy in the galaxy cluster Abell 3827 (Massey et al. 2015). The dynamical origin of this offset and its long-lived nature are not well-understood (see Kuhlen et al. 2013). Massey et al. (2015) claim that the offset seen in a cluster galaxy could be due to an astrophysical effect or could be evidence for self-interacting dark matter. A lower resolution may only give an upper limit to the offset; for example, Schaller et al. (2015) find that more than $95 \%$ of the simulated galaxies in their sample have an offset between the stars and dark matter halo that is smaller than the gravitational softening length of $700 \mathrm{pc}$ they use. The dynamics of an offset halo in a disk could well have some similarities with the unsettled behaviour seen in central regions of galaxies (Miller \& Smith 1992). Such "sloshing" or unrelaxed central region is seen in advanced mergers as well as in isolated galaxies (Jog \& Maybhate 2006), though this phenomenon has received little attention.

In this paper we study how the spatial distribution and the kinematics in the central disk are affected by the off-center dark matter halo, in a region where the disk is the dominant mass component. We do this in terms of a simple, idealized analytical model. The halo potential can be written in terms of its value with respect to the disk center and a perturbation which takes a lopsided form. Next, the equations of motion in the disk plane are solved under this joint disk and halo system. We find that the offset halo results in a surprisingly large disturbance over the central few kpc. This effect is strongly dependent on the radius. For typical exponential disk and pseudo-isothermal halo parameters for the Milky Way, and assuming an offset of $350 \mathrm{pc}$ as seen by Kuhlen et al. (2013) in their simulations, the resulting radial variation in the orbit is $\sim 40 \%$ at $R=1.5 \mathrm{kpc}$ which drops down to $\sim 3 \%$ at $3 \mathrm{kpc}$. The kinematics is also strongly perturbed. Thus, even a small offset of $350 \mathrm{pc}$ can induce significant spatial and kinematical lopsidedness in the central $\leq 3 \mathrm{kpc}$ region.

Section 2 contains the formulation of equations and Sect. 3 contains the results. Sections 4 and 5 contain the discussion and conclusion, respectively.

\section{Formulation of equations}

We use cylindrical co-ordinates $(R, \phi, z)$. The disk is taken to have an exponential surface density distribution (Freeman 1970), and the dark matter halo is taken to have a screened or 


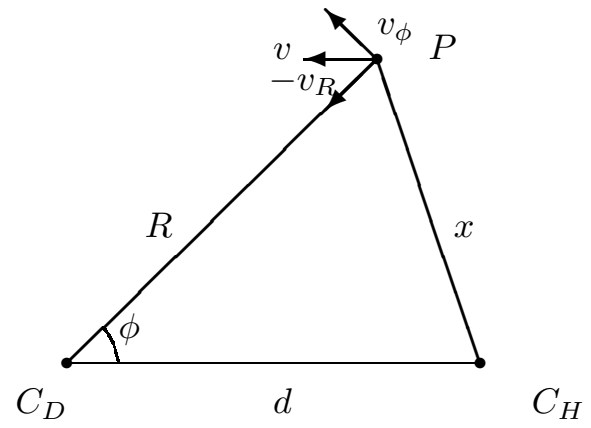

Fig. 1. Schematic diagram showing the centers of disk and an off-center dark matter halo, separated by a distance $d$, and their effect at point $P$ in the disk plane.

pseudo-isothermal spherical density profile (Binney \& Tremaine 1987). The disk is assumed to be razor-thin, and the dark matter halo center is assumed to be in the same plane as the galactic disk plane, for simplicity. Thus, we only treat the planar disturbance arising due to the halo offset.

\subsection{Potential due to a disk and off-center halo}

The potential at a point $P$ in the galactic disk plane due to the disk and the off-center halo can be written in terms of the disk and halo contributions with respect to the disk center, plus the additional perturbation term which arises due to the offset between the disk and the halo centers. We show later that this additional term is indeed a perturbation. This follows from the fact that in a typical spiral galaxy like our Galaxy, the disk dominates the halo dynamics in the central region.

The disk center and the halo center are denoted by $C_{\mathrm{D}}$ and $C_{\mathrm{H}}$, and the offset between the two is denoted by a distance $d$. The schematic diagram is given in Fig. 1. Here $\phi$ is the angle between the radius vector to the point $P$ from the disk center and the direction between the disk center and the halo center. We note that the figure is not drawn to scale, where for the sake of clarity we have shown $d \sim R$, whereas in our calculation we assume $d \ll R$.

The density distribution of an exponential disk and a pseudoisothermal halo are given respectively as

$\Sigma(R)=\Sigma_{0} \exp \left(-\frac{R}{R_{\mathrm{D}}}\right)$

and

$\rho(r)=\frac{\rho_{0}}{1+\frac{r^{2}}{R_{\mathrm{C}}^{2}}}$

where $r$ is given in spherical co-ordinates. Here $\Sigma_{0}$ is the central surface density of the disk and $R_{\mathrm{D}}$ is the disk scale-length; $\rho_{0}$ is the central density of the halo and $R_{\mathrm{C}}$ is the halo core radius.

Solving the Poisson equation, the potential in the mid-plane at a point $P$ due to the disk is given by (see, e.g., Binney \& Tremaine 1987)

$\Phi_{\mathrm{D}}=-\pi G \Sigma_{0} R\left[I_{0}(y) K_{1}(y)-I_{1}(y) K_{0}(y)\right]$,

where $y=R / 2 R_{\mathrm{D}}$; and $I_{n}$ and $K_{n}(n=0,1)$ are modified Bessel functions of the first and second kind, respectively.
The halo mid-plane potential at the point $P$ when the halo is concentric with respect to the disk is obtained as

$$
\begin{aligned}
\Phi_{\mathrm{H}}(d=0)= & 4 \pi G \rho_{0} R_{\mathrm{C}}^{2}\left[\frac{\log \left(R_{\mathrm{C}}^{2}+R^{2}\right)}{2}\right. \\
& \left.+\frac{R_{\mathrm{C}}}{R} \arctan \left(\frac{R}{R_{\mathrm{C}}}\right)-1\right] .
\end{aligned}
$$

The halo mid-plane potential at the point $P$ when the halo is offcenter is given by

$\Phi_{\mathrm{H}}=4 \pi G \rho_{0} R_{\mathrm{C}}^{2}\left[\frac{\log \left(R_{\mathrm{C}}^{2}+x^{2}\right)}{2}+\frac{R_{\mathrm{C}}}{x} \arctan \left(\frac{x}{R_{\mathrm{C}}}\right)-1\right]$.

We consider small values of $d(\ll R)$, so that

$x^{2}=R^{2}+d^{2}-2 d R \cos \phi \approx R^{2}\left(1-\frac{2 d \cos \phi}{R}\right)$.

Using this expression, the first two terms in Eq. (5) are simplified separately as

$$
\begin{aligned}
& \log \left(R_{\mathrm{C}}^{2}+x^{2}\right) \approx \log \left(R_{\mathrm{C}}^{2}+R^{2}\right)-\frac{2 d R \cos \phi}{R_{\mathrm{C}}^{2}+R^{2}} \\
& \frac{R_{\mathrm{C}}}{x} \arctan \left(\frac{x}{R_{\mathrm{C}}}\right) \approx \frac{R_{\mathrm{C}}}{R} \arctan \left(\frac{R}{R_{\mathrm{C}}}\right) \\
&+\frac{d R_{\mathrm{C}} \cos \phi}{R^{2}} \arctan \left(\frac{R}{R_{\mathrm{C}}}\right)
\end{aligned}
$$

Therefore, the first terms from both expressions are simply terms from a halo that is concentric with the disk, and the other two terms arise from the non-zero value of $d$. Thus, the total halo potential, $\Phi_{\mathrm{H}}$ is given by

$$
\begin{aligned}
\Phi_{\mathrm{H}}= & \Phi_{\mathrm{H}}(d=0)+4 \pi G \rho_{0} R_{\mathrm{C}}^{2} d \cos \phi \\
& \times\left[\frac{R_{\mathrm{C}}}{R^{2}} \arctan \left(\frac{R}{R_{\mathrm{C}}}\right)-\frac{R}{R_{\mathrm{C}}^{2}+R^{2}}\right],
\end{aligned}
$$

where the second term on the right-hand side defines the perturbation term $\Phi_{\text {pert }}$. Thus, the total potential can be written as

$\Phi=\Phi_{\mathrm{D}}+\Phi_{\mathrm{H}}(d=0)+\Phi_{\text {pert }}$,

where the first two terms give the potential due to disk and halo with the same center, $C_{\mathrm{D}}$, and the last term is the perturbation potential due to the halo being offset with respect to the disk center.

\subsection{Disk response to lopsided perturbation potential}

The perturbation potential (see Eq. (9)) is thus given by

$\Phi_{\text {pert }}(R, \phi)=\Psi(R) \cos \phi$,

where

$\Psi(R)=4 \pi G \rho_{0} R_{\mathrm{C}}^{2} d\left(\frac{R_{\mathrm{C}} \arctan \frac{R}{R_{\mathrm{C}}}}{R^{2}}-\frac{R}{R_{\mathrm{C}}^{2}+R^{2}}\right)$.

The perturbation potential depends linearly on $\cos \phi$, thus it has a lopsided form corresponding to an $m=1$ azimuthal perturbation (e.g., Jog \& Combes 2009). This is as expected from the geometry of the mass distribution (see the schematic plot in Fig. 1). Its magnitude is linearly proportional to the separation $d$ between 
the disk and the halo centers. Thus, as the offset $d \rightarrow 0$, the perturbation goes to zero and the net potential is then simply the sum of the potentials due to the disk and a concentric halo.

Next we define $\epsilon_{\text {pert }}$, the dimensionless perturbation potential, to be the ratio of the perturbation potential over the total undisturbed contributions of concentric disk and halo:

$\epsilon_{\mathrm{pert}}(R)=\frac{\Psi(R)}{\Phi_{\mathrm{D}}+\left[\Phi_{\mathrm{H}}(d=0)\right]}$.

Using $\Psi(R)$ as the perturbation potential, and following the procedure for linear perturbation calculations as in Jog (1997, 2000), we next derive the perturbed radius and the azimuthal and radial velocity components for a particle in a closed circular orbit of radius $R_{0}$.

The perturbation in $R$ is thus obtained as

$\delta R=-\left(\frac{2 \Psi}{R}+\frac{\partial \Psi}{\partial R}\right)_{R_{0}} \frac{\cos \phi_{0}}{\kappa^{2}-\Omega_{0}^{2}}$,

where $\kappa$ and $\Omega_{0}$ denote the epicyclic frequency and the angular speed, respectively, at $R_{0}$. Thus,

$\delta R=-\frac{d \cos \phi_{0}}{\kappa^{2}-\Omega_{0}^{2}} \frac{4 \pi G \rho_{0} R_{\mathrm{C}}^{2}}{R_{\mathrm{C}}^{2}+R_{0}^{2}}\left[\frac{R_{\mathrm{C}}^{2}}{R_{0}^{2}}+\frac{2 R_{0}^{2}}{R_{\mathrm{C}}^{2}+R_{0}^{2}}-3\right]$.

The perturbation in the azimuthal velocity $v_{\phi}$ is governed by

$R_{0} \frac{\mathrm{d}^{2} \delta \phi}{\mathrm{d} t^{2}}+2 \Omega_{0} \frac{\mathrm{d} \delta R}{\mathrm{~d} t}=\frac{\Psi \sin \phi_{0}}{R_{0}}$.

Integrating with respect to time, this gives

$R_{0} \frac{\mathrm{d} \delta \phi}{\mathrm{d} t}=-2 \Omega_{0} \delta R-\frac{\Psi(R) \cos \phi_{0}}{\Omega_{0} R_{0}}$.

The net velocity along the azimuthal direction is given by

$v_{\phi}=v_{\mathrm{c}}+R_{0} \frac{\mathrm{d} \delta \phi}{\mathrm{d} t}+\Omega_{0} \delta R$.

Here $v_{\mathrm{c}}$ is the unperturbed rotational velocity. The last two terms give $\delta v_{\phi}$, the perturbed velocity along the azimuthal direction, and using Eq. (17) this is

$\delta v_{\phi}=-\left(\frac{\Psi \cos \phi_{0}}{\Omega_{0} R_{0}}+\Omega_{0} \delta R\right)$.

In full this is given as

$$
\begin{aligned}
\delta v_{\phi}= & -\frac{d \cos \phi_{0}}{\Omega_{0}} 4 \pi G \rho_{0} R_{\mathrm{C}}^{2}\left[\frac{R_{\mathrm{C}} \arctan \left(\frac{R_{0}}{R_{\mathrm{C}}}\right)}{R_{0}^{3}}-\frac{1}{R_{0}^{2}+R_{\mathrm{C}}^{2}}\right] \\
& +\frac{d \cos \phi_{0} \Omega_{0}}{\kappa^{2}-\Omega_{0}^{2}} \frac{4 \pi G \rho_{0} R_{\mathrm{C}}^{2}}{R_{0}^{2}+R_{\mathrm{C}}^{2}}\left[\frac{R_{\mathrm{C}}^{2}}{R_{0}^{2}}+\frac{2 R_{0}^{2}}{R_{0}^{2}+R_{\mathrm{C}}^{2}}-3\right] .
\end{aligned}
$$

The perturbed radial velocity is given by

$v_{R}=\frac{\mathrm{d} R}{\mathrm{~d} t}=\frac{\mathrm{d}\left(R_{0}+\delta R\right)}{\mathrm{d} t}$.

Using Eq. (15), this is given as

$v_{R}=\frac{d \sin \phi_{0}}{\kappa^{2}-\Omega_{0}^{2}} \frac{4 \pi G \Omega_{0} \rho_{0} R_{\mathrm{C}}^{2}}{R_{\mathrm{C}}^{2}+R_{0}^{2}}\left[\frac{R_{\mathrm{C}}^{2}}{R_{0}^{2}}+\frac{2 R_{0}^{2}}{R_{\mathrm{C}}^{2}+R_{0}^{2}}-3\right]$.

While discussing the results in Sect. 3 we refer to the radius as $R$ for convenience (without the subscript 0 ).

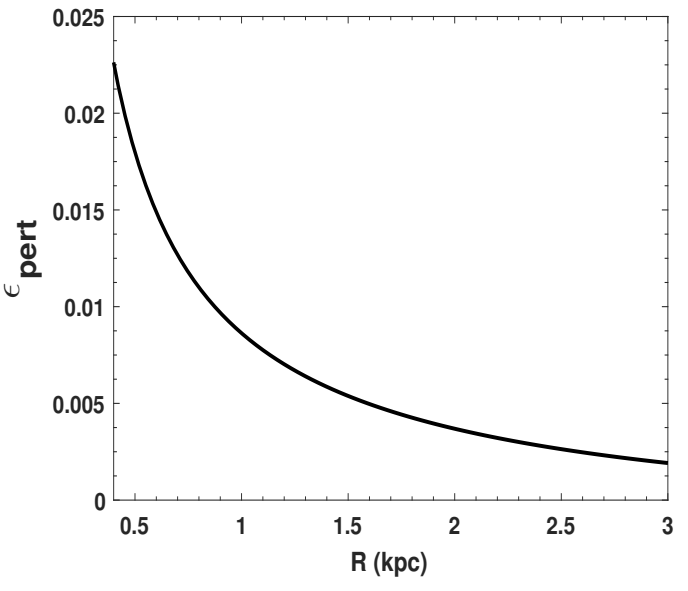

Fig. 2. Perturbation potential $\epsilon_{\text {pert }}$ arising due to the halo that is offcenter by $350 \mathrm{pc}$ with respect to the disk versus the disk radius $R$.

\section{Results}

\subsection{Input parameters}

The above results are general, and are valid for a typical spiral galaxy where the disk dominates the dynamics in the central region. We now consider the input parameters for the Galaxy and study the effect of the off-center halo on the central disk region. We take the central disk surface density, $\Sigma_{0}=640.9 M_{\odot} \mathrm{pc}^{-2}$, and the exponential disk scale-length, $R_{\mathrm{D}}=3.2 \mathrm{kpc}$; and the halo central density, $\rho_{0}=0.035 M_{\odot} \mathrm{pc}^{-3}$, and the core radius, $R_{\mathrm{c}}=5 \mathrm{kpc}$; these values are taken from the Galaxy mass model by Mera et al. (1998).

\subsection{Resulting disk lopsidedness}

First, we plot the value of the dimensionless perturbation potential, $\epsilon_{\text {pert }}(R)$, defined in Eq. (13) for a choice of $d=350 \mathrm{pc}$ (see Fig. 2).

We check that $\epsilon_{\mathrm{pert}}(R)$ is indeed a perturbation, justifying the assumption made in Sect. 2.1. Its value increases as $R$ decreases, which is expected physically, since the perturbative effect of the halo would be stronger at a smaller radius. Thus, the magnitude of the perturbation potential falls with increasing radius $R$. By $R=1 \mathrm{kpc}$, the fractional value of the perturbation potential is less than $1 \%$. Yet, as we show below, this still results in strong spatial and kinematical lopsidedness of the closed orbits in the disk, because the perturbed equations have a strong dependence on radius.

In Fig. 3a we plot the net radius of the orbit vs. $\phi$ for an initial circular orbit of radius $R=1.5 \mathrm{kpc}$. The values for the other half of the azimuthal range are not shown as these would give a mirror image of this plot, since these quantities depend simply on $\cos \phi$. Figure $3 \mathrm{~b}$ shows a polar plot of the perturbed orbit drawn against unperturbed, original circular orbits at $R_{0}=1$, $2 \mathrm{kpc}$. Thus the perturbed orbit shows a typical lopsided form: it is shortened along $\phi=0^{\circ}$ by $\sim 40 \%$ and elongated along the opposite direction (i.e., along $\phi=180^{\circ}$ ). The net azimuthal and radial velocity components vs. $\phi$ are shown in Figs. $3 c$ and d, respectively. We note that the change in azimuthal velocity is huge: its maximum magnitude (which occurs at $\phi=0^{\circ}$ ) is $72.2 \mathrm{~km} \mathrm{~s}^{-1}$. The resulting radial or streaming velocity value, which occurs at $\phi=90^{\circ}$, is also significant $=35.2 \mathrm{~km} \mathrm{~s}^{-1}$. Thus, the radius and the velocity components of the perturbed orbit are strongly 

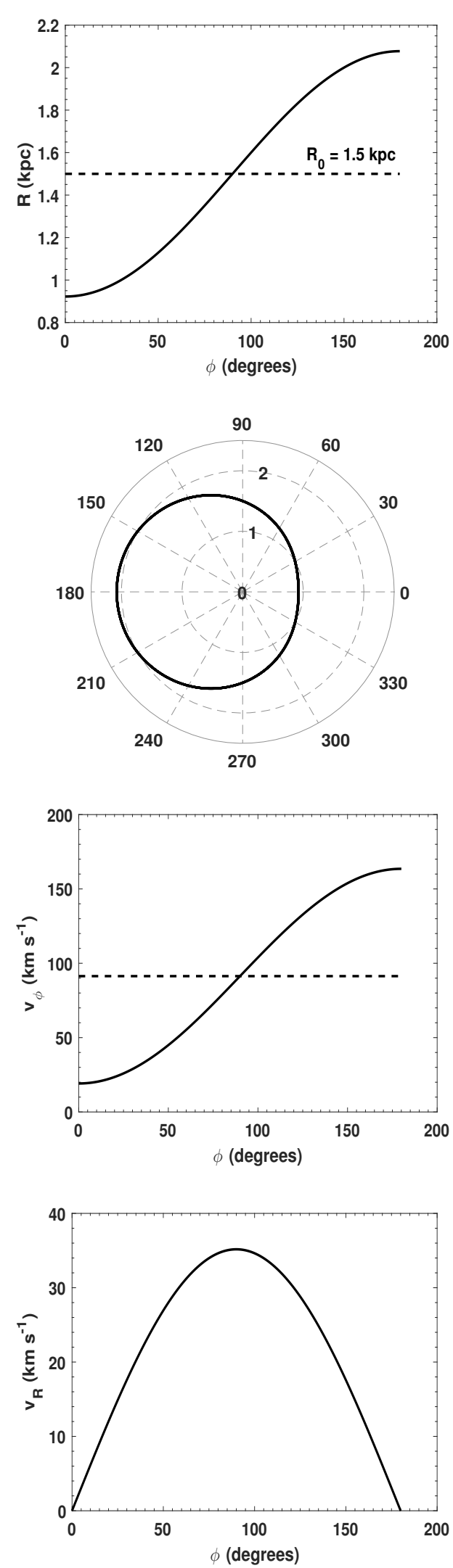

Fig. 3. Resulting orbit for an initial circular orbit at $R=1.5 \mathrm{kpc}$, shown as $R$ vs. $\phi$ in panel a), and as a polar plot in panel b). The net azimuthal and radial velocity components vs. $\phi$ are shown in panels c) and d), respectively. The dashed line in panel c) denotes $v_{\mathrm{c}}$. Panels a) and b) show that the orbit is strongly distorted or lopsided spatially: it is shortened along $\phi=0^{\circ}$ and elongated in the opposite direction, i.e., along $\phi=180^{\circ}$.

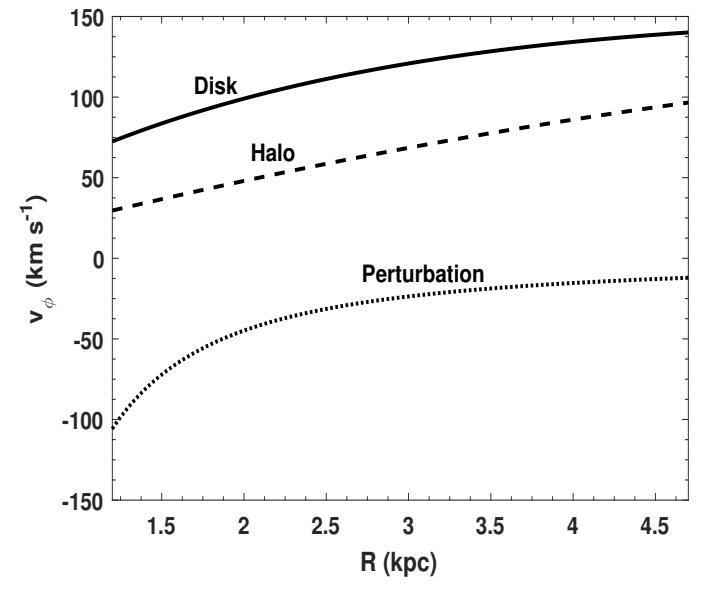

Fig. 4. Azimuthal velocity components vs. the radius $R$ for the diskalone and the halo, both with the same center; and the perturbed azimuthal velocity component, along $\phi=0^{\circ}$, arising when the halo is taken to be offset with respect to the disk center by $350 \mathrm{pc}$. We note that the disk dominates the dynamics in the central few kpc, and the perturbed velocity component is large.

lopsided due to the perturbation potential resulting from the offcenter halo.

The resulting strong lopsidedness is further illustrated in Fig. 4 where the azimuthal velocity due to concentric disk and halo, as well as as the perturbation value, all obtained at $\phi=0^{\circ}$ are shown as a function of $R$. The net unperturbed velocity is given by the square root of the disk and halo contributions added in quadrature. The perturbation in azimuthal velocity has a negative sign with respect to the rotation, and its magnitude increases rapidly below $1.5 \mathrm{kpc}$, and can be comparable to the original rotational velocity. These features indicate that the perturbed orbit is no longer a nearly circular orbit, rather it takes on the shape of a radial orbit.

The fractional values of the change in the radius and the azimuthal velocity for the perturbed motion with respect to the undisturbed values are shown in Figs. 5a and b, respectively. Clearly a significant radial range up to $R=3 \mathrm{kpc}$ is strongly disturbed, as can be seen from the spatial and kinematical lopsidedness. In fact, the assumption of linear perturbation in the calculations in Sect. 2 is not strictly valid below about $R<1.2 \mathrm{kpc}$. Hence, in Figs. 4 and 5 we have only shown results beyond this radius. We note that at larger radii (beyond $3.2 \mathrm{kpc}$ ), the sign of the radial change is opposite so that it is positive at $\phi=0^{\circ}$; however, the magnitude of the fractional change is very small (see Fig. 5a).

We next check the dependence of the results on the input parameters. First we vary the halo concentration by taking $R_{\mathrm{c}}=$ $4 \mathrm{kpc}$ and $6 \mathrm{kpc}$ (instead of $5 \mathrm{kpc}$ as was used above); these correspond respectively to a more centrally concentrated and low mass halo, and a more extended and massive halo. As expected, a lower core radius leads to a lower magnitude of the perturbation potential, $\epsilon_{\text {pert }}$. The resulting fractional variation in the orbital radius at $R=1.5 \mathrm{kpc}$ is now $20 \%$ for $R_{\mathrm{c}}=4 \mathrm{kpc}$ as opposed to $40 \%$ obtained for $R_{\mathrm{c}}=5 \mathrm{kpc}$, while a higher $R_{\mathrm{c}}=6 \mathrm{kpc}$ results in a higher relative fractional change in radius of $62 \%$. Since the perturbation potential as well as the resulting radial and velocity changes are linearly proportional to the offset value $d$, a value of $d$ smaller than $350 \mathrm{pc}$ that was used above will lead to correspondingly smaller perturbed values.

As shown above, an off-center dark matter halo leads to strong central disk lopsidedness. This is in contrast to the fairly 

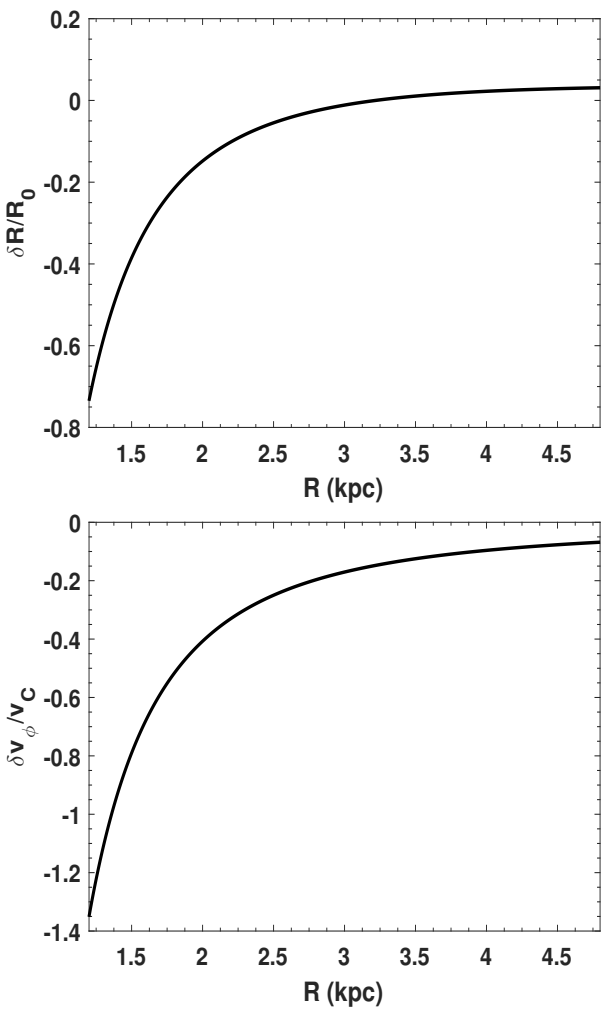

Fig. 5. Fractional change in the radius of the orbit (top panel) and fractional change in the azimuthal velocity component (bottom panel) both obtained at $\phi=0^{\circ}$ vs. the radius $R$.

moderate lopsidedness seen in the outer disks of galaxies (Rix \& Zaritsky 1995; Jog 1997). The outer disks of galaxies are often observed to be lopsided where a radially constant perturbation potential gives a good fit to the observations (Rix \& Zaritsky 1995; Angiras et al. 2007; Zaritsky et al. 2013). In that case, at $\phi=0^{\circ}$, the typical fractional change in the radius is equal to twice the fractional perturbation potential (i.e., about $10 \%$; Jog 2000); and the fractional change in the azimuthal velocity is equal to the fractional perturbation potential (i.e., about 5\%; Jog 2002). In contrast, for the off-center halo considered here, the resulting spatial and velocity perturbations have much higher values and show a strong radial dependence. This occurs because here the perturbed equations have a strong dependence on radius and do not give a simple linear dependence on the dimensionless perturbation potential $\epsilon_{\text {pert }}$.

\section{Discussion}

We next discuss a few issues related to this work.

(1). We have treated a kinematic response of the disk to the offset halo for simplicity. A more realistic model would involve taking account of the back-reaction of the induced disk lopsidedness on the halo. We defer such a complex, selfconsistent calculation to a future paper.

(2). We have shown that in a typical spiral galaxy like our Galaxy, the dark matter halo that is offset by $350 \mathrm{pc}$ with respect to the galactic disk center disturbs the orbits and kinematics substantially within the central few kpc of the disk. Since the offset is known to be long-lived from simulation studies (Kuhlen et al. 2013), the resulting disk lopsidedness will also be long-lived.
This can have far-reaching implications for the evolution of the central region. For example, the resulting lopsided orbits may be important in feeding the active galactic nucleus (AGN) at the center. The highly disturbed, lopsided orbits in the central region could lead to an outward angular momentum transport, thus helping gas infall, similar to what was shown for lopsidedness in the outer disk (Saha \& Jog 2014), and in agreement with the infall proposed for the central region (Combes 2016). We note that this process could be important in fueling a central AGN even in an isolated galaxy, which is a long-standing problem.

(3). We have assumed the initial orbit to be circular. However, when the random velocity dispersion is taken into account, the particle executes an epicyclic motion around this radius, hence the true undisturbed motion is not circular, and also not closed (since $\kappa / \Omega$ is in general an irrational number). Incidentally, this situation also occurs in the case of lopsidedness in the outer disk (Rix \& Zaritsky 1995; Jog 1997). The net disturbed motion then can be thought of as the circular motion, plus the superposition of the epicyclic motion and the perturbed motion due to the lopsided potential.

The random velocity dispersion is known to be high in the galactic center region. The observations by Lewis \& Freeman (1989) show that the stellar radial velocity in the Galactic disk has a central value of $95 \mathrm{~km} \mathrm{~s}^{-1}$ and falls exponentially with radius with a scale-length of $8.7 \mathrm{kpc}$. This gives the disk velocity dispersion of $80 \mathrm{~km} \mathrm{~s}^{-1}$ at $R=1.5 \mathrm{kpc}$. This is comparable to the rotational velocity at this radius of $91 \mathrm{~km} \mathrm{~s}^{-1}$, obtained by adding the disk and halo contributions in quadrature, taken from Fig. 4. This figure also gives $\kappa=111 \mathrm{~km} \mathrm{~s}^{-1} \mathrm{kpc}^{-1}$. Thus at this radius, the radial amplitude of the lopsided motion is $0.6 \mathrm{kpc}$ (Fig. 5a), while the epicyclic amplitude is $=80 \mathrm{~km} \mathrm{~s}^{-1} / 111 \mathrm{~km} \mathrm{~s}^{-1} \mathrm{kpc}^{-1}(\sim 0.7 \mathrm{kpc})$, so the two are comparable. A similar case occurs in the outer galactic disk where the two perturbations lead to comparable radial amplitudes, and yet the epicyclic amplitude is ignored for the sake of simplicity (Rix \& Zaritsky 1995; Jog 1997). A similar simplification is made in the treatment of a stellar disk perturbed by the triaxial halo (Bailin et al. 2007). In the present paper also, we only take into account the lopsided perturbed motion with respect to the circular motion. Thus, our model is simple and idealized but it does still bring out the result of strong disk lopsidedness arising due to the dark matter halo offset.

(4). Generally, once an $m=1$ feature develops, it is long-lived, lasting for several Gyr, as has been shown for the lopsidedness in the outer galactic disk (Ideta 2002; Saha et al. 2007; also see Jog \& Combes 2009). The self-gravity of the disk reduces the differential precession, and that leads to this long life. A similar process would operate in the central region, thus the $m=1$ configuration in the disk arising due to halo offset could be long-lived. This issue needs to be addressed by future $N$-body simulations.

\section{Conclusion}

We study the effect of an off-center dark matter halo on the central galactic disk, where the disk dominates the dynamics. To do this, we develop a simple, analytical model where the equations of motion are formulated in terms of the potential contributions of the disk and halo when these two are concentric, plus a perturbation term that arises due to the halo offset. We show that the perturbation potential has a lopsided form, i.e., it shows an 
$m=1$ azimuthal dependence. The halo offset thus acts as a lopsided perturbation potential, and we study the disk response to this. The magnitude of the perturbation potential depends on the halo parameters and also the offset distance $d$ between the disk and the halo centers. Though the fractional perturbation potential is small $(<1 \%)$, we find that it has a surprisingly strong effect on the orbits and the kinematics in the central region within a radius of about $3 \mathrm{kpc}$. The resulting strong disk lopsidedness can have important dynamical consequences, for example it can help fuel the central AGN via an outward angular momentum transfer. An off-center dark matter halo thus results in complex and interesting dynamics in the central region that should be explored in future work.

Acknowledgements. We would like to thank the anonymous referee for thoughtful comments which have greatly improved the paper. C.J. would like to thank the DST, Government of India for support via a J.C. Bose fellowship (SB/S2/JCB-31/2014).

\section{References}

Angiras, R. A., Jog, C. J., Dwarakanath, K. S., \& Verheijen, M. A. W. 2007, MNRAS, 378, 276

Bailin, J., Simon, J. D., Bolatto, A. D., Gibson, B. K., \& Power, C. 2007, ApJ, 667,191
Binney, J., \& Tremaine, S. 1987, Galactic Dynamics (Princeton: Princeton University Press)

Chemin, L., Hure, J.-M., Soubiran, C., et al. 2016, A\&A, 588, A48

Combes, F. 2016, in IAU Symp. 322, eds. S. Longmore, G. Bicknell, \& R Crocker, 245

Freeman, K. C. 1970, ApJ, 160, 811

Ideta, M. 2002, ApJ, 568, 190

Jog, C. J. 1997, ApJ, 488, 642

Jog, C. J. 2000, ApJ, 542, 216

Jog, C. J. 2002, A\&A, 391, 47

Jog, C. J., \& Combes 2009, Phys. Rep., 471, 75

Jog, C. J., \& Maybhate, A. 2006, MNRAS, 370, 891

Klypin, A., Zhao, H., \& Somerville, R. S. 2002, ApJ, 573, 597

Kuhlen, M., Guedes, J., Pillepich, A., Madau, P., \& Mayer, L. 2013, ApJ, 765, 10

Lewis, J. R., \& Freeman, K. C. 1989, AJ, 97, 139

Massey, R., Williams, L., Smit, R., et al. 2015, MNRAS, 449, 3393

Mera, D., Chabrier, G., \& Schaeffer, R. 1998, A\&A, 330, 953

Miller, R. H., \& Smith, B. F. 1992, ApJ, 393, 508

Rix, H.-W., \& Zaritsky, D. 1995, ApJ, 447, 82

Rubin, V. 1983, Science, 220, 1339

Sackett, P. 1997, ApJ, 483, 103

Saha, K., \& Jog, C. J. 2014, MNRAS, 444, 352

Saha, K., Combes, F., \& Jog, C. J. 2007, MNRAS, 382, 419

Schaller, M., Robertson, A., Massey, R., Bower, R. G., \& Eke, V. R. 2015, MNRAS, 453, L58

Zaritsky, D., Salo, H., \& Laurikainen, E. et al. 2013, ApJ, 772, 135 